\title{
Are Doppler ultrasonography parameters symmetric between the right and left kidney?
}

This article was published in the following Dove Press journal:

International Journal of General Medicine

15 November 2010

Number of times this article has been viewed

\author{
Khalil Ansarin' \\ Abolhassan Shakeri Bavil ${ }^{2}$ \\ Kamyar Ghabili ${ }^{3}$ \\ Mohammadali M Shoja ${ }^{4}$ \\ Hamid T Khosroshahi ${ }^{5}$ \\ Babak Hajipour ${ }^{6}$ \\ R Shane Tubbs ${ }^{7}$ \\ Mojtaba Parvizi ${ }^{8}$ \\ 'Tuberculosis and Lung Disease \\ Research Center, Tabriz University \\ of Medical Sciences, Tabriz, Iran; \\ ${ }^{2}$ Department of Radiology, Tabriz \\ University of Medical Sciences, Tabriz, \\ Iran; 'Students' Research Committee, \\ Tabriz University of Medical Sciences, \\ Tabriz, Iran; ${ }^{4}$ Indiana University \\ Department of Neurosurgery, \\ Indianapolis, IN, USA; ${ }^{5}$ Department \\ of Nephrology, Tabriz University \\ of Medical Sciences, Tabriz, Iran; \\ ${ }^{6}$ Faculty of Medicine, Tabriz University \\ of Medical Sciences, Tabriz, Iran; \\ ${ }^{7}$ Section of Pedriatric Neurosurgery, \\ Children's Hospital, Birmingham, AL, \\ USA; ${ }^{8}$ Department of Pathology and \\ Medical Biology, University Medical \\ Center Groningen, Groningen, The \\ Netherlands
}

Background: Among numerous modalities applied for evaluation of kidney diseases, Doppler ultrasonography (DU) provides information about the hemodynamic status of the kidneys. Meanwhile, the variability in DU parameters of the right and left kidney is a matter of controversy. The aim of this study was to determine whether any difference exists between the DU indices of the right and left kidney.

Methods: Retrospectively, we collected DU findings of 25 healthy potential renal transplant donors. All donors underwent renal DU and multidetector computed tomographic angiography before donor nephrectomy. DU indices, including peak systolic volume (PSV), resistive index (RI), pulsatility index (PI), end-diastolic volume (EDV), and acceleration time (AT), were recorded.

Results: The median age of the donors was 27 (range 23-39) years. The median PSV, RI, $\mathrm{EDV}$, and AT for the right kidney were $29 \mathrm{~cm} / \mathrm{sec}, 0.59,10.9 \mathrm{~cm} / \mathrm{sec}$, and $50 \mathrm{msec}$, respectively. For the left kidney, the median PSV, RI, EDV, and AT were, respectively, $26.8 \mathrm{~cm} / \mathrm{sec}, 0.60$, $10.6 \mathrm{~cm} / \mathrm{sec}$, and $43 \mathrm{msec}$. Among the DU indices, median PI of the right kidney was significantly different from that of the left kidney (1.02 versus $0.95, P=0.01)$.

Conclusion: In conclusion, the present study revealed that right kidney DU indices, except for PI, may not differ from those of the left kidney.

Keywords: Doppler ultrasonography, peak systolic volume, resistive index, pulsatility index, kidney

\section{Introduction}

Among the numerous modalities used for evaluation of kidney diseases, renal Doppler ultrasonography (DU) provides information about the hemodynamic status of the kidneys. ${ }^{1,2}$ Owing to the repeatability of this technique, any temporal alterations within the renal arteries due to treatment protocols can be easily followed by DU indices, including peak systolic velocity (PSV), pulsatility index (PI), and resistive index $(\mathrm{RI}){ }^{2,3}$ Alterations in these parameters have been noted in a range of conditions affecting the kidney, such as acute variations in renal vascular resistance (eg, renal artery stenosis) and renal damage in multiple-organ dysfunction syndrome. ${ }^{4,5}$ For instance, diagnosis of renal artery stenosis is based on the presence of asymmetry in renal blood flow detected with imaging modalities, including DU. ${ }^{6}$ However, a few studies with controversial results have investigated whether any asymmetry in DU parameters exists between the right and left kidney. ${ }^{7-10}$ The aim of this study was to determine whether any difference exists between the DU indices of the right and left kidney in healthy individuals.
Correspondence: Kamyar Ghabili Students' Research Committee, Tabriz University of Medical Sciences, Tabriz, Iran $\mathrm{Tel}+989144106136$

Fax +98 4II 336 II 20

Email kghabili@gmail.com 


\section{Methods}

In a retrospective study, we collected DU findings of 25 healthy potential renal transplant donors from October 2004 to July 2008. All these potential donors underwent renal DU of the interlobar and arcuate arteries in the superior and inferior lobes and middle part of the kidneys. The average values of DU indices were recorded thereafter. DU indices, including peak systolic volume (PSV), resistive index (RI), pulsatility index (PI), end-diastolic volume (EDV), and acceleration time (AT) were measured and recorded by a radiologist using an Hitachi EUB.525 ultrasound machine (Hitachi Medical Corp., Tokyo, Japan). RI was calculated using built-in software as follows: RI = [PSV-EDV]/PSV. Prior to donor nephrectomy, computed tomographic angiography (CTA) was performed by multidetector computed tomography (Somatom Sensation 64, Siemens, Germany). Six donors were excluded from the present study due to the presence of supernumerary renal artery detected by CTA. Data were presented as median (interquartile range). All statistical analyses were performed with Statistical Package for Social Sciences (SPSS Inc., Chicago, IL, USA) for Windows version 16.0. The Wilcoxon signed rank test was used to compare the DU indices between the right and left kidney. A $P$ value $<0.05$ was considered statistically significant.

\section{Results}

Twenty-five renal transplant donors were included, of median age 27 years (range 23-39 years, including two women and 23 men). The median PSV, RI, EDV, and AT for the right kidney were $29 \mathrm{~cm} / \mathrm{sec}$ (24.3-36.2), 0.59 (0.53-0.62), $10.9 \mathrm{~cm} / \mathrm{sec}(9.4-15.05)$, and $50 \mathrm{msec}$ (43-64), respectively. For the left kidney, the median PSV, RI, EDV, and AT were, respectively, $26.8 \mathrm{~cm} / \mathrm{sec}$ (25.1-33.35), 0.60 (0.55-0.63), $10.6 \mathrm{~cm} / \mathrm{sec}(9.6-14.6)$, and $43 \mathrm{msec}$ (39-57). Among the DU indices, median PI of the right kidney was significantly different from that of the left kidney (1.02 (0.90-1.15) versus $0.95(0.86-1.09), P=0.01$, Wilcoxon signed rank test (see Table 1)).

Table I Doppler ultrasonographic indices of the healthy individuals' right and left kidneys $(n=25)$

\begin{tabular}{llll}
\hline & Right kidney & Left kidney & $\begin{array}{l}\text { P value } \\
\text { (two-tailed) }\end{array}$ \\
\hline PSV $(\mathrm{cm} / \mathrm{sec})$ & $29(24.3-36.2)$ & $26.8(25.10-33.35)$ & 0.876 \\
PI & $1.02(0.90-1.15)$ & $0.95(0.86-1.09)$ & $0.016^{\dagger}$ \\
RI & $0.59(0.53-0.62)$ & $0.60(0.55-0.63)$ & 0.604 \\
EDV $(\mathrm{cm} / \mathrm{sec})$ & $10.9(9.4-15.05)$ & $10.6(9.6-14.6)$ & 0.783 \\
AT $(\mathrm{msec})$ & $50(43-64)$ & $43(39-57)$ & 0.075 \\
\hline
\end{tabular}

Notes: "Wilcoxon signed rank test; 'statistically significant $(P<0.05)$.

Abbreviations: PSV, peak systolic velocity; PI, pulsatility index; RI, resistive index; EDV, end-diastolic velocity; AT, acceleration time.

\section{Discussion}

The results of this study showed that DU indices, excluding PI, did not vary between the right and left kidney in healthy individuals. This is in agreement with the findings of Milovanceva-Popovska and Dzikova, ${ }^{7}$ Keogan et al, ${ }^{8}$ and Murat et $\mathrm{al}^{11}$ that failed to detect any difference in RI between the kidneys. On the other hand, no remarkable variation in $\mathrm{RI}$ and PI of both kidneys has been reported within canine and feline renal arteries. ${ }^{12}$ Interestingly, Yildirim et al found no inequality in flow velocity waveform indices, including PSV, EDV, RI, and PI of the renal arteries on both sides in the neonatal population. ${ }^{9}$ In contrast with our findings, Kliewer et al revealed PSV, among the DU parameters for early systole, as a varying parameter between the kidneys. ${ }^{10}$ However, they concluded that such asymmetry in PSV was clinically insignificant. ${ }^{10}$ In the present study, although not compelling, PI of the right kidney was higher than that of the left kidney. However, we do not have an explanation for such a difference in PI values between the two kidneys.

The concept of anatomic, physiologic, and functional asymmetry between the kidneys has been a matter of interest for researchers. A number of clinicians recommend that functional asymmetry of the kidneys should be investigated preoperatively to determine which kidney should be donated and transplanted. ${ }^{13,14}$ In addition, considerable differences in renal blood flow (RBF) have been reported between the right and left kidneys in some previous investigations. Peters et al estimated lower RBF in the right than in the left kidney in normal subjects. ${ }^{15}$ On the other hand, substantial differences between left and right RBF have been reported in groups of hypertensive patients. ${ }^{6,16-18}$ Because DU is widely used to evaluate RBF in patients with renal disease, a probable asymmetry in DU parameters, similar to that in RBF, between two kidneys might be hypothesized. Nonetheless, we could not identify any asymmetry in DU parameters, except for PI, between the two kidneys.

In conclusion, the present study revealed that right kidney DU indices, except for PI, may not differ from those of the left kidney. Further investigations with a larger sample size are needed to assess DU parameters between the right and left kidneys.

\section{Acknowledgment}

This study was financially supported by a grant from the Students' Research Committee, Research Vice Chancellor's Office of the Tabriz University of Medical Sciences. 


\section{Disclosure}

The authors report no conflicts of interest in this work, which was presented as a poster at the 11th Congress of the Middle East Society for Organ Transplantation, November 2008, Shiraz, Iran.

\section{References}

1. Lin ZY, Dai CY, Chang WY, et al. Influence of posture change on intrarenal arterial resistive index measurement. Abdom Imaging. 2002;27:626-628.

2. Ghabili K, Khosroshahi HT, Shakeri A, et al. Can Doppler ultrasonographic indices of the renal artery predict the presence of supernumerary renal arteries? Transplant Proc. 2009;41:2731-2733.

3. Duranteau J, Deruddre S, Vigue B, et al. Doppler monitoring of renal hemodynamics: Why the best is yet to come. Intensive Care Med. 2008;34:1360-1361.

4. Sugiura T, Nakamori A, Wada A, et al. Evaluation of tubulointerstitial injury by Doppler ultrasonography in glomerular diseases. Clin Nephrol. 2004;61:119-126.

5. Ardalan MR, Shoja MM, Tubbs RS, et al. Transplant renal artery stenosis associated with acute cytomegalovirus infection: resolution following ganciclovir administration. Ren Fail. 2009;31:982-984.

6. van Onna M, Houben AJ, Kroon AA, et al. Asymmetry of renal blood flow in patients with moderate to severe hypertension. Hypertension. 2003;41:108-113.

7. Milovanceva-Popovska M, Dzikova S. Doppler ultrasonography: A tool for nephrologists - single centre experience. Prilozi. 2008;29:107-128

8. Keogan MT, Kliewer MA, Hertzberg BS, et al. Renal resistive indexes: Variability in Doppler US measurement in a healthy population. Radiology. 1996;199:165-169.
9. Yildirim H, Gungor S, Cihangiroglu MM, et al. Doppler studies in normal kidneys of preterm and term neonates: Changes in relation to gestational age and birth weight. J Ultrasound Med. 2005;24:623-627.

10. Kliewer MA, Hertzberg BS, Keogan MT, et al. Early systole in the healthy kidney: Variability of Doppler US waveform parameters. Radiology. 1997;205:109-113.

11. Murat A, Akarsu S, Ozdemir H, et al. Renal resistive index in healthy children. Eur J Radiol. 2005;53:67-71.

12. Novellas R, Espada Y, Ruiz de Gopegui R. Doppler ultrasonographic estimation of renal and ocular resistive and pulsatility indices in normal dogs and cats. Vet Radiol Ultrasound. 2007;48:69-73.

13. Oh CK, Yoon SN, Lee BM, et al. Routine screening for the functional asymmetry of potential kidney donors. Transplant Proc. 2006;38:1971-1973.

14. Shokeir AA, Gad HM, Shaaban AA, et al. Differential kidney scans in preoperative evaluation of kidney donors. Transplant Proc. 1993;25:2327-2329.

15. Peters AM, Gunasekera RD, Henderson BL, et al. Noninvasive measurement of blood flow and extraction fraction. Nucl Med Commun 1987;8:823-837.

16. Kioschos JM, Kirkendall WM, Valenca MR, et al. Unilateral renal hemodynamics and characteristics of dye-dilution curves in patients with essential hypertension and renal disease. Circulation. 1967;35:229-249.

17. Baldwin DS, Hulet WH, Biggs AW, et al. Renal function in the separate kidneys of man. II. Hemodynamics and excretion of solute and water in essential hypertension. J Clin Invest. 1960;39:395-404.

18. van Jaarsveld BC, Krijnen P, Derkx FH, et al. The place of renal scintigraphy in the diagnosis of renal artery stenosis. Fifteen years of clinical experience. Arch Intern Med. 1997;157:1226-1234.
International Journal of General Medicine

\section{Publish your work in this journal}

The International Journal of General Medicine is an international, peer-reviewed open-access journal that focuses on general and internal medicine, pathogenesis, epidemiology, diagnosis, monitoring and treatment protocols. The journal is characterized by the rapid reporting of reviews, original research and clinical studies across all disease areas.

\section{Dovepress}

A key focus is the elucidation of disease processes and management protocols resulting in improved outcomes for the patient.The manuscript management system is completely online and includes a very quick and fair peer-review system. Visit http://www.dovepress.com/ testimonials.php to read real quotes from published authors. 